\title{
A DTL WITH SHORT TANKS AND EXTERNAL FOCUSING FOR HIGH POWER CW LINACS
}

\author{
C. Bourat, C. Perraudin \\ Thomson-CSF Airsys, Bagneux,-France-
}

\section{Abstract}

Recently, major projects, for the transmutation of nuclear waste, the production of tritium or more generally for the production of neutrons by spallation, have been proposed using high power linacs and a CW operation. See for example [1] or [2]. In this context, the manufacturing of the low or medium energy part of the linac with a standard DTL, appears to be a tricky job, particularly the insertion of high gradient quadrupoles into the drift tubes, with a specific cooling system needed for $\mathrm{CW}$ operation, and many brazing or welding processes. Moreover the reliability and the maintenance of the DTL could be affected sooner or later. It is proposed in this paper to use a DTL with short tanks ( let say 2 to 5 cells ) with a focusing system outside the tanks. The mechanical design is in that way very simplified, the drift tube geometry can be optimised for a better shunt impedance, the cooling system is easy and efficient and the alignment problem is greatly reduced. This concept is applied to a $5 \mathrm{MeV}$ to 100 $\mathrm{MeV} \mathrm{CW}$ proton linac operating at $352 \mathrm{MHz}$, with a 40 $\mathrm{mA}$ beam current, and shows moreover that the linac global efficiency is increased compared with a classical approach. Preliminary beam dynamics studies are included.

\section{INTRODUCTION}

A classical DTL could generally be divided in long Alvarez-type tanks, with a FODO focusing system integrated inside the drift tubes. As part of the design of

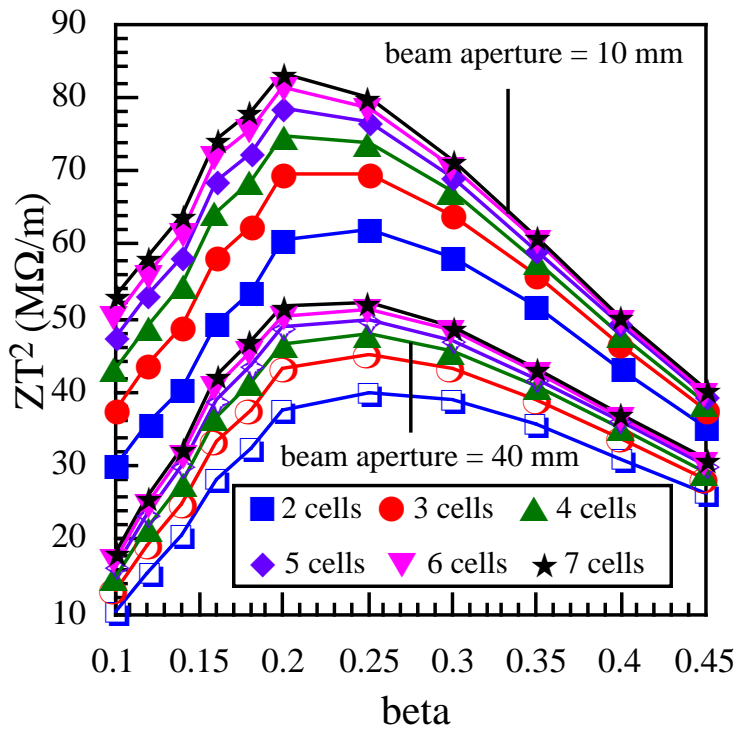

Fig.1 Shunt impedance (100\%) for multi-cells tanks (352 MHz). Bore radius $=\phi 10 \mathrm{~mm}, \phi 20 \mathrm{~mm}, \phi 40 \mathrm{~mm}$ new high power proton linacs operating in a $\mathrm{CW}$ mode, the cavities cooling system is thus an important task to take into account. It must be designed to ensure the removal of heat due to high RF wall losses on drift tubes and quadrupole heating at once. This is especially difficult at low energy where the drift tubes are short and the quadrupole gradient could be high. In that case the bore diameter should be moreover minimised, adding a restriction to the beam dimensions. The manufacturing of such drift tubes, including a compact quadrupole, its power feeding with cooled wires, the cooling circuit for the drift tube itself, and associated with the stem, implies in particular many multi-stages brazing and welding processes which are significative in terms of risks and costs. To date, two solutions among others have been proposed to move the focusing system outside the tanks:

- the Coupled-Cavity DTL ( CCDTL) developed at Los Alamos [3], designed to work at $700 \mathrm{MHz}$ for the APT linac.

- the Separated-type DTL ( SDTL ) from KEK [4], [2] at $432 \mathrm{MHz}$, for medium proton energies between 150 $\mathrm{MeV}$ and $300 \mathrm{MeV}$

\section{A SDTL FOR LOW ENERGY PROTON BEAM}

It is proposed here to apply the SDTL concept at 352 $\mathrm{MHz}$ [1], with a CW operating mode, for low proton energies from say $5 \mathrm{MeV}$ to $100 \mathrm{MeV}$, just behind an RFQ for example, in place of a conventional DTL.

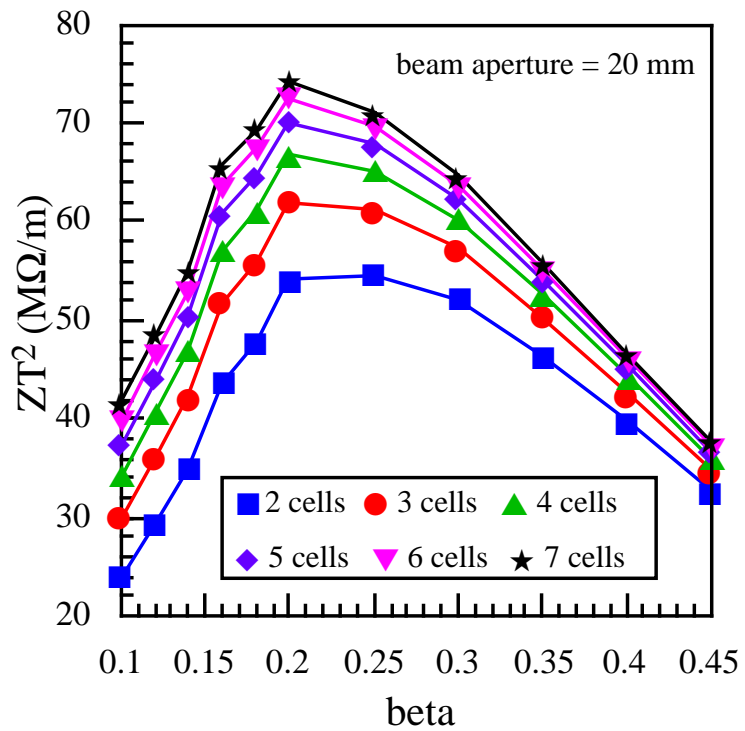


The tanks are necessarily short, with few cells to not lengthen too much the transverse focusing period. They are not RF coupled together, but are fed independently with a power splitting scheme, according to the power that couplers can handle. Several potential advantages, previously mentioned elsewhere, could be pointed out:

- a mechanical simplification and efficient cooling of the drift tubes, including its inner part exposed potentially to the beam

- a better shunt impedance with an optimised geometry, or alternatively a greater beam aperture if desired.

- post-couplers in short tanks no longer needed

- lower manufacturing costs

The overall SDTL has the following properties:

- easier to align with a magnetic axis separated from the RF one.

- diagnostics could be set up between tanks.

- a manufacturing simplicity implies in principle a better reliability and maintenance.

\subsection{The SDTL Shunt Impedance}

No quadrupoles inside the drift tubes allows to optimise its geometry to find the best shunt impedance. The outer drift tube radius is minimised, leading to a light and compact tube, including exclusively a cooling circuit. See for example fig. 2. The gap of the cell is optimised for a maximum $\mathrm{ZT}^{2}$. Two key points must be kept in mind:

- the limited number of cells implies that the power losses in end walls have to be taken into account for the global shunt impedance of the tank.

- the reduced size of the drift tube, associated with a short gap, could lead to a high peak field on copper.

This maximum field is here arbitrarily limited to about 1 Kilpatrick ( i.e. $18.4 \mathrm{MV} / \mathrm{m}$ at $352 \mathrm{MHz}$ ) which is enough conservative to avoid sparking. This restriction applies to tanks below $16 \mathrm{MeV}$, which are thus nonoptimised for maximum shunt impedance, with a gap larger than expected.

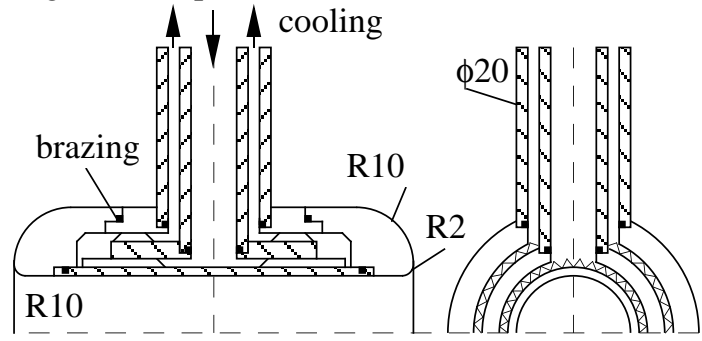

Fig.2 A SDTL Drift Tube with cooling system

Superfish [5] computations are made with various beam apertures from $\phi 10 \mathrm{~mm}$ to $\phi 40 \mathrm{~mm}$, versus the particle velocity from $\beta=0.1$ ( $4.7 \mathrm{MeV}$ ) to $\beta=0.45$ ( $112.4 \mathrm{MeV}$ ) and the global $\mathrm{ZT}^{2}$ is thus estimated for multi-cells tanks from 2 to 7 cells. ( Fig. 1 ). The maximum shunt impedance is obtained around $20-30 \mathrm{MeV}$, which justifies the use of a SDTL at low energy. Compared with a conventional DTL with a same bore radius, a $40 \%$ to $70 \%$ increase in $\mathrm{ZT}^{2}$ could be gained particularly above 15 $\mathrm{MeV}$.

\subsection{A $5 \mathrm{MeV}$ to $100 \mathrm{MeV}$ SDTL Linac}

The design of the linac includes at the beginning some beam dynamics constraints. To limit the longitudinal phase advance without current $\sigma_{\mathrm{ol}}$ below $90 \mathrm{deg}$, on account of beam stability, short tanks with 2 cells are used at low energy, with an RF power level restricted to $62.5 \mathrm{~kW}$ max, which is also compatible with the maximum field on copper. The number of cells is gradually increased up to 5 cells at $23 \mathrm{MeV}$. The transitions in number of cells are smoothed by choosing specific laws of synchronous phase, while keeping a regular and constant RF power splitting scheme ( $1 / 16$ and $1 / 8$ of $1 \mathrm{MW}$ klystrons ). The design is an iterative process between the beam dynamics, the parameters of the tanks and the choice of transitions.

The solution proposed here includes a drop of $17.5 \%$ on the theoretical shunt impedance, as a safety margin. The beam current is $40 \mathrm{~mA} \mathrm{CW}$. The beam aperture is kept constant at $20 \mathrm{~mm}$. The parameters are the following:

- 8 klystrons ( $1 \mathrm{MW}, 352 \mathrm{MHz}$ ) of which the two first are divided by 16 ( $62.5 \mathrm{~kW}$ per tank ), and others divided by 8 ( $125 \mathrm{~kW}$ per tank )

- 80 tanks, $336 \beta \lambda$ cells, divided as follows: - 2 cells ( tanks 1 to $12,5 \mathrm{MeV}$ to $8.8 \mathrm{MeV}$, $\phi_{\mathrm{s}}=48 \mathrm{deg}$ to $52 \mathrm{deg}$ )

- 3 cells ( tanks 13 to $20,8.8 \mathrm{MeV}$ to $13.5 \mathrm{MeV}$, $\left.\phi_{\mathrm{s}}=40 \mathrm{deg}\right)$

- 4 cells ( tanks 21 to $32,13.5 \mathrm{MeV}$ to $23 \mathrm{MeV}$, $\phi_{\mathrm{s}}=35 \mathrm{deg}$ to $\left.45 \mathrm{deg}\right)$

- 5 cells ( tanks 33 to $80,23 \mathrm{MeV}$ to 103.2 $\mathrm{MeV}, \phi_{\mathrm{s}}=25 \mathrm{deg}$ to $30 \mathrm{deg}$, up to tank 40 , and $\phi_{\mathrm{s}}=30$ deg beyond )

- active length $=83.36 \mathrm{~m}$

- average field $\mathrm{E}_{\mathrm{o}} \mathrm{T}=1.44 \mathrm{MV} / \mathrm{m}$

- average shunt impedance $\mathrm{ZT}^{2}=42.6 \mathrm{M} \Omega / \mathrm{m}$

- copper power losses $=4.07 \mathrm{MW}$

- beam power $=3.93 \mathrm{MW}$

- efficiency $=49.1 \%$

The high energy part of the SDTL beyond $20 \mathrm{MeV}$, because of its high shunt impedance, compensate largely the low energy part which is not designed at its potential maximum capability, due to the transitions and large synchronous phase ( which however increases the phase acceptance ). This SDTL example shows in fact a gain of at least $10 \%$ in efficiency compared with a standard DTL which could be designed even with a smaller beam aperture. The SDTL saves 1 or 2 klystron power units.

The overall linac uses here a doublet focusing scheme which is well suited to the set up of free space between the quadrupoles for various beam diagnostics. Here, a regular $\beta \lambda$ drift is used. In that case the total length of the linac is $124 \mathrm{~m}$ ( about 1.5 times the active length ) which 
is also $20 \%$ or $30 \%$ longer than a standard DTL. A triplet focusing could also be used, leading to a longer linac, but with the advantage of a round beam in the cavities. Fig 3 shows the main parameters of the beam dynamics for an initial $40 \mathrm{~mA}$ matched beam, with an ellipsoidal uniform space charge model and 5 times the RMS beam emittance at the output of an RFQ before the SDTL. ( i.e. $\varepsilon_{\mathrm{tn}}=1.2$ $\pi$ mm.mrad normalised, and $\varepsilon_{1}=0.6 \mathrm{MeV} . \mathrm{deg}$ )

\section{CONCLUDING REMARKS}

The SDTL scheme presented here seems to be an attractive solution for a low energy high power linac operating in the $\mathrm{CW}$ mode, especially for its manufacturing simplicity and its potential low cost. It uses moreover an independently-phased cavities scheme, well known in superconducting linacs [6], which must be favourable for larger velocity acceptance. Nevertheless, a great care must be taken for the beam dynamics at low energy, because of the transitions and the matching to the RFQ [7]. Other

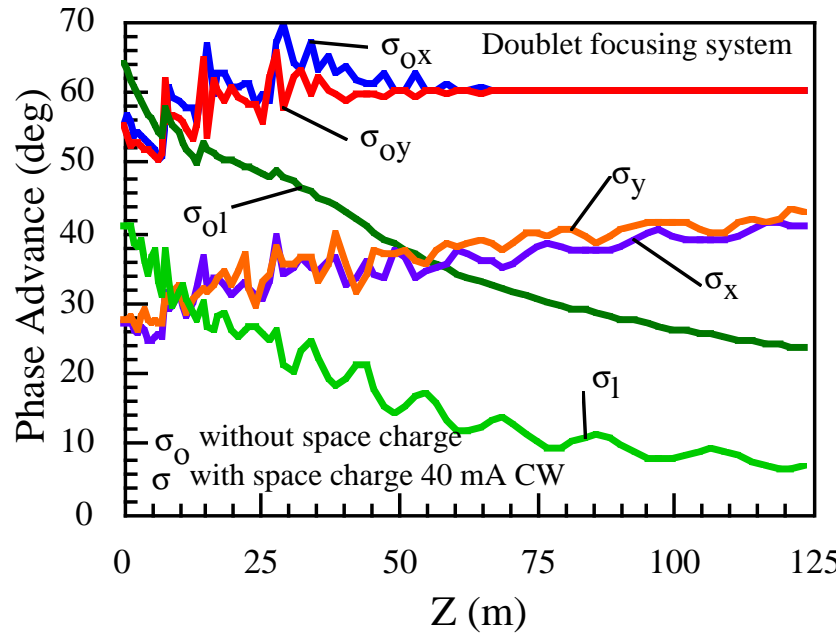

lay-out schemes are now under investigation for a smoother beam behaviour.

\section{REFERENCES}

[1] M. Promé, "Major Projects for the use of High Power Linacs", LINAC-96, CERN 96-07, p. 9

[2] Y. Yamazaki, "Design Issues for High-Intensity, High-Energy Proton Accelerators", LINAC-96, CERN 96-07, p. 592

[3] J.H. Billen and al. "A New RF Structure for Intermediate-Velocity Particles", LINAC-94, Tsukuba,p. 341

[4] T. Kato, "Proposal of a Separated-type Proton Drift Tube Linac for a medium-energy Structure", KEK internal report 92-10

[5] K. Halbach, R.F. Holsinger, Particle Accelerators 1976 Vol. 7, pp 213-222

[6] K.C.D. Chan, "Conceptual Design of a Superconducting HighIntensity Proton Linac", LINAC-96, CERN 96-07, p. 580

[7] J.H. Billen, H. Takeda, L.M. Young, "Smooth Transverse and Longitudinal Focusing in High-Intensity Ion Linacs", LINAC-96, CERN 96-07, p. 587

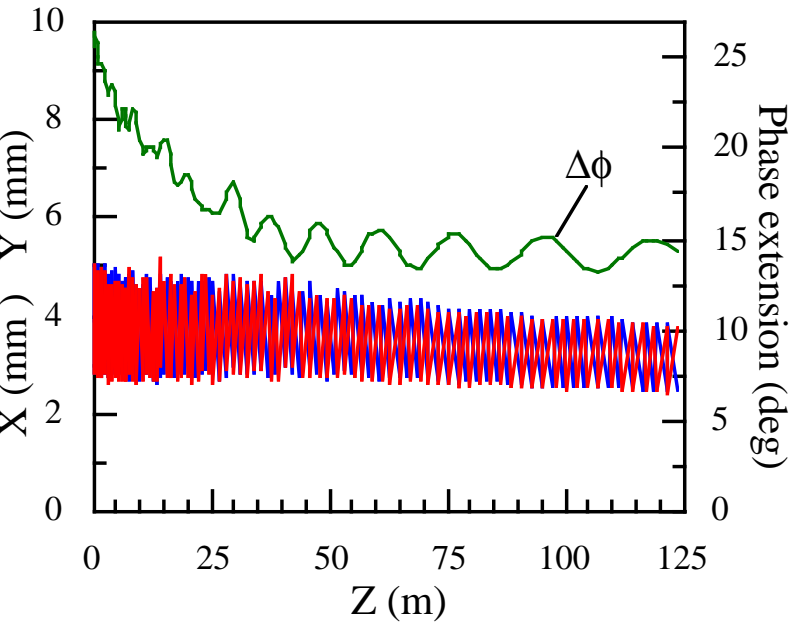

Fig. 3 Phase advance laws along the SDTL, Transverse beam envelopes and Phase extension

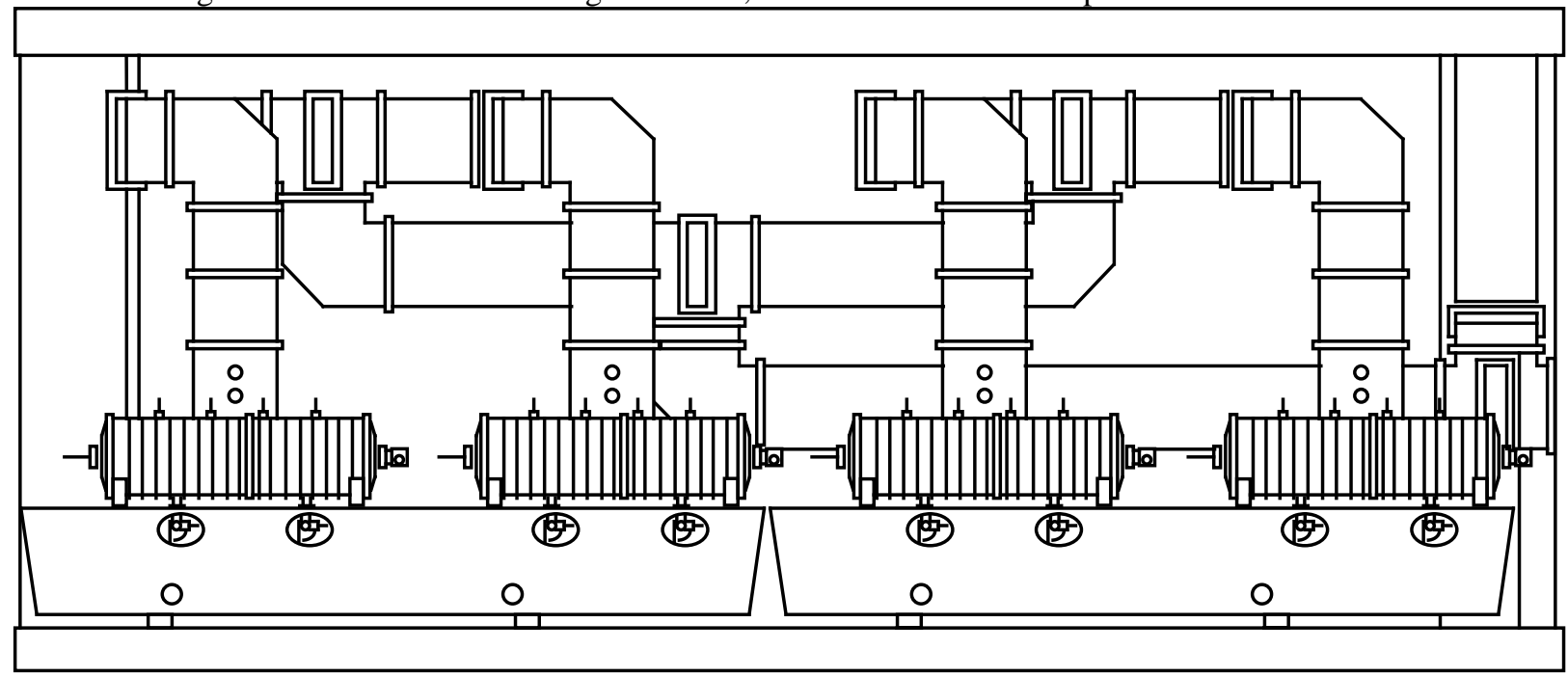

Fig.4 Lay-out of a $100 \mathrm{MeV}, 5$ cells SDTL ( $125 \mathrm{~kW}$ per tank, 1/2 klystron, $352 \mathrm{MHz}$ ) 